\title{
1,3-Bis[tert-butyl(di-tert-butylfluorsilyl)amino]trisulfan - eine Verbindung mit FSiNSSSNSiF-Helix
}

\author{
Uwe Klingebiel *, Frank Pauer, George M. Sheldrick und Dietmar Stalke
}

Institut für Anorganische Chemie der Universität Göttingen,

Tammannstraße 4, W-3400 Göttingen

Eingegangen am 5. Juni 1991

Key Words: Disulfane, bis[(fluorosilyl)amino] derivative / Trisulfane, 1,3-bis[(fluorosilyl)amino] derivative / Selane, bis[(fluorosilyl)amino] derivative / Helix structure

\section{1,3-Bis[tert-butyl(di-tert-butylfluorosilyl)amino]trisulfane - a Compound with FSiNSSSNSiF Helix}

The lithium derivative of tert-butyl(di-tert-butylfluorosilyl)amine (1) reacts with sulfur or selenium to give the bis[(fluorosilyl)-amino]di- and -trisulfanes 2, 3 and -selane 4, respectively. The trisulfane 3 crystallizes from $n$-hexane and exhibits a helical structure (X-ray analysis).
$N$-Silylierte Aminosulfane sind sowohl durch Reaktion von Schwefel als auch von Chlorsulfanen mit Silylamiden zugänglich $^{1)}$. Bis[bis(trimethylsilyl)amino]selan und -tellan wurden kürzlich aus Lithium-bis(trimethylsilyl)amid und Dichlordiselan bzw. $\mathrm{TeCl}_{4}$ hergestellt und kristallstrukturanalytisch untersucht ${ }^{2}$. Ebenfalls bekannt ist die entsprechende Schwefelverbindung ${ }^{3}$.

Unbekannt sind bisher Reaktionen von Chalkogenen mit lithiierten Aminofluorsilanen. Diese Verbindungen zeigen sowohl das Reaktionsverhalten von Silylamiden als auch von Iminosilanen (Schema 1) ${ }^{4-6)}$.

Schema 1

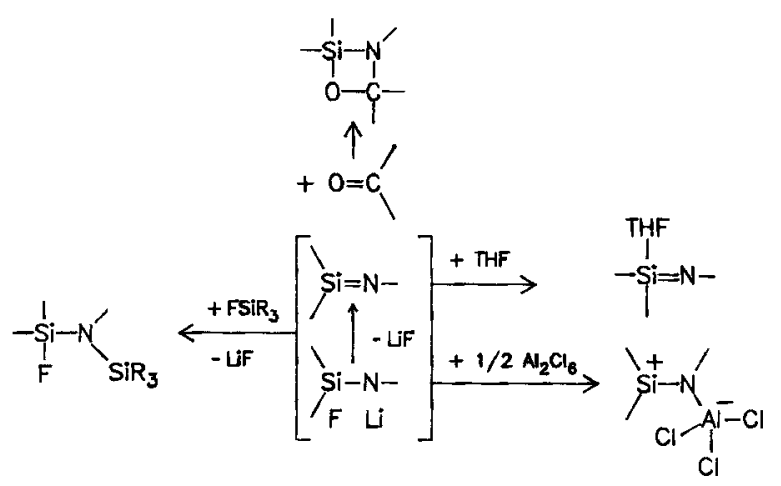

Reaktionen mit Aldehyden, Ketonen ${ }^{5)}, \mathrm{Al}_{2} \mathrm{Cl}_{6}{ }^{4)}, \mathrm{THF}^{6}$ ) führen zu Verbindungen, die als Produkte von intermediär gebildeten Iminosilanen mit den Reaktanden gedeutet werden können.

Im vorliegenden Beitrag beschreiben wir das Reaktionsverhalten des Lithium-Derivats von Di-tert-butyl(tert-butylamino)fluorsilan (1) gegenüber Schwefel und Selen. Da Iminophosphane mit Chalkogenen unter Koordinationsaufweitung reagieren, schlossen wir die vergleichbare $\mathrm{Re}$ aktion eines intermediären Iminosilans nicht völlig aus. Jedoch stellten wir fest, daß 1 mit Schwefel und auch Selen als Amid reagiert, d.h. das Chalkogen wird nucleophil unter Abbau des $\mathbf{S}_{8}$-Ringes bzw. der $\mathrm{Se}_{n}$-Ketten angegriffen. Es entstehen die Bis[(fluorsilyl)amino]sulfane 2, 3 und das Selan 4. Während auch in unterschiedlich molaren Ansätzen als Reaktionsprodukte von 1 mit $S_{8}$ Bis(fluorsilylamino)di(2) und -trisulfan (3) isoliert wurden, bildete sich mit Selen ausschließlich das Monoselan 4 (Schema 2). Die große Reaktivität von 1 ermöglichte erstmals den Einsatz von elementarem Selen bei der Synthese eines (Silylamino)selans.

Schema 2

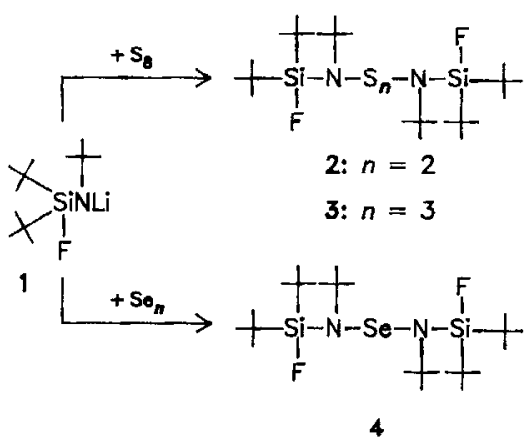

2-4 destillieren im Vakuum unzersetzt. 4 ist bei Raumtemperatur rot und zähflüssig. 3 kristallisiert nach der Destillation aus.

Im Kristall ordnet sich die FSiNS $_{3}$ NSiF-Kette in einer Helix an (Abb. 1). Die Bindungslängen entsprechen weitgehend den Summen der Kovalenzradien der beteiligten Atome. Die Si-F-Bindungen haben Werte von 160.0 und $160.8 \mathrm{pm}(\Sigma r k=160.7 \mathrm{pm})$; die Werte der Si-N-Bindungen betragen 175.1 bzw. $175.4 \mathrm{pm}(\Sigma r k=175.3 \mathrm{pm})$, die $\mathrm{S}-\mathrm{N}$-Abstände sind mit 166.8 und 168.0 pm etwas kürzer als der berechnete Wert $(171.5 \mathrm{pm})$. Andererseits sind die S-S-Bindungen (210.2 und $208.2 \mathrm{pm}$ ) marginal länger als der berechnete Wert $(208.0 \mathrm{pm})^{7}$.

Nach der VSEPR-Theorie sollte der S-S-S-Winkel etwas kleiner als der Tetraederwinkel sein. Dies trifft mit 
$104.7^{\circ}$ zu. Die N-Atome $\left(\Sigma \mathrm{N} 1: 359.3^{\circ}, \Sigma \mathrm{N} 2: 359.8^{\circ}\right)$ besitzen eine planare Umgebung. Die $\mathrm{S}-\mathrm{S}-\mathrm{N}$-Winkel $(110.0$ und $110.3^{\circ}$ ) sind gegenüber dem Tetraederwert leicht aufgeweitet.
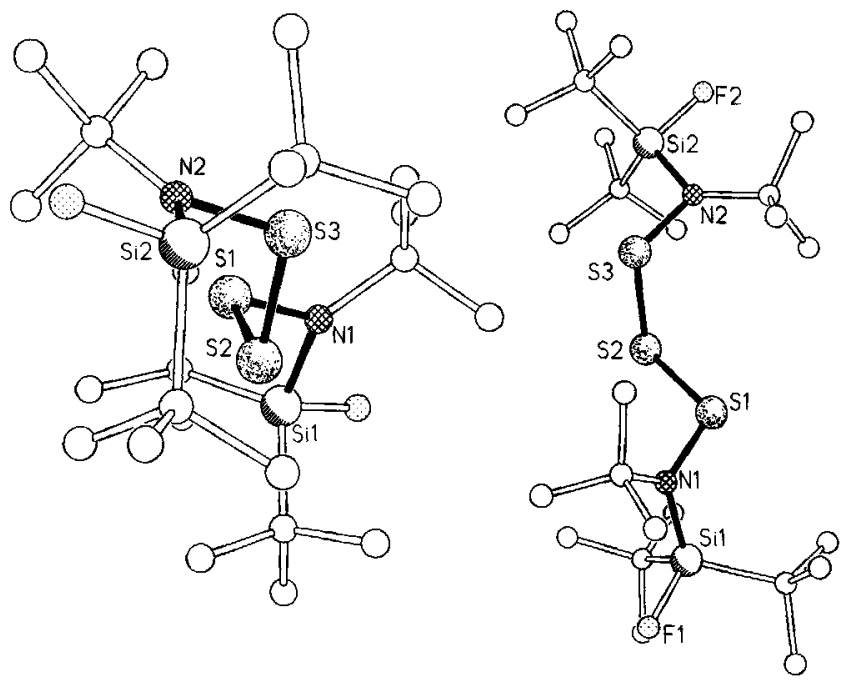

Abb. 1. Struktur von $3 \mathrm{im}$ Kristall; ausgewählte Bindungslängen [pm] und -winkel $\left[{ }^{\circ}\right]$ : S (1) - S(2) 210.2(1), S(1)-N(1) 166.8(2), $\mathrm{S}(2)-\mathrm{S}(3) \quad 208.2(1), \mathrm{S}(3)-\mathrm{N}(2) \quad 168.0(2), \mathrm{N}(1)-\mathrm{Si}(1) \quad 175.1(2)$, $\mathrm{Si}(1)-\mathrm{F}(1) \quad 160.8(2), \quad \mathrm{N}(2)-\mathrm{Si}(2) \quad 175.4(2), \quad \mathrm{Si}(2)-\mathrm{F}(2) \quad 160.0(2) ;$ $\mathrm{S}(2)-\mathrm{S}(1)-\mathrm{N}(1) 110.0(1), \mathrm{S}(1)-\mathrm{S}(2)-\mathrm{S}(3)$ 104.7(1), $\mathrm{S}(2)-\mathrm{S}(3)-$ $\mathrm{N}(2) 110.3(1), \mathrm{S}(1)-\mathrm{N}(1)-\mathrm{Si}(1) 107.5(1), \mathrm{N}(1)-\mathrm{Si}(1)-\mathrm{F}(1)$ 103.4(1), $\mathrm{S}(3)-\mathrm{N}(2)-\mathrm{Si}(2) 107.8(1), \mathrm{N}(2)-\mathrm{Si}(2)-\mathrm{F}(2) 105.2(1)$

Für die Unterstützung dieser Arbeit danken wir der Deutschen Forschungsgemeinschaft und dem Fonds der Chemischen Industrie.

\section{Experimenteller Teil}

Strukturbestimmung von 3: Datensammlung bei $-120^{\circ} \mathrm{C}$ auf einem STOE-Siemens-AED-Diffraktometer, graphitmonochromatisierte Mo-K $\mathrm{K}_{\alpha}$-Strahlung ( $\left.\lambda=71.073 \mathrm{pm}\right)$. Strukturlösung mit Direkten Methoden ${ }^{8)}$, Kleinste-Quadrate-Rechnungen. Anisotrope Verfeinerung der Nichtwasserstoffatome, H-Atome geometrisch ideal positioniert und mit festen Auslenkungsparametern nach dem Reitermodell $(d \mathrm{CH}=98 \mathrm{pm})$ verfeinert. $\eta$-Verfeinerung: $\eta=$ 1.1(1).

$\mathrm{C}_{24} \mathrm{H}_{54} \mathrm{~F}_{2} \mathrm{~N}_{2} \mathrm{~S}_{3} \mathrm{Si}_{2}$, Molmasse $561.1 \mathrm{~g} \mathrm{~mol}^{-1}$; orthorhombische Raumgruppe $P 2{ }_{1}{ }_{1}{ }_{2}{ }_{1} ; a=894.6(1), b=1572.2(1), c=2319.8(2)$ pm; $V=3.263 \mathrm{~nm}^{3} ; Z=4 ; \mathrm{Q}_{\text {ber }}=1.155 \mathrm{Mgm}^{-3} ; \mu=0.32 \mathrm{~mm}^{-1}$; STOE-Vierkreisdiffraktometer AED, Mo-K,$T=-120^{\circ} \mathrm{C}$; Profiloptimierte $2 \Theta-\omega$-Abtastungen; Kristallgröße $0.4 \times 0.4 \times 0.4$ $\mathrm{mm}$; 3706 Reflexe bis $2 \Theta_{\max }=45^{\circ}$ gemessen, davon 3260 symmetrieunabhängige und mit $\left|F_{0}\right|>3 \sigma\left(\left|F_{0}\right|\right)$ beobachtete Reflexe $\boldsymbol{m}=3131$; verfeinerte Parameter $n=298$; Übereinstimmungsgüte $\left[\Sigma \omega\left(\left|F_{\mathrm{o}}\right|-\left|F_{\mathrm{c}}\right|\right)^{2} /(m-n)\right]^{0.5}=1.51 ; \quad R=\Sigma|| F_{\mathrm{o}}|-| F_{\mathrm{c}}|| / \Sigma\left|F_{\mathrm{o}}\right|=$ $0.0264 ; \omega R=R_{\mathrm{g}}=\left[\Sigma \omega\left(\left|F_{\mathrm{o}}\right|-\left|F_{\mathrm{c}}\right|\right)^{2} / \Sigma \omega\left|F_{\mathrm{o}}\right|^{2}\right]^{0.5}=0.0325 ;$ Wichtungsschema $\omega^{-1}=\sigma^{2}\left|F_{0}\right|+0.0002\left|F_{0}\right|^{2}$; Restelektronendichte $\left[10^{6} \mathrm{e}-\mathrm{pm}^{-3}\right]:$ Max $/ \mathrm{min}$ 2.7/2.0; Programm SHELXS-86 ${ }^{8}$, SHELX$76^{9}$. Tab. 1 enthält die Atomparameter und U(eq)-Werte.

Weitere Einzelheiten zur Kristallstrukturanalyse können beim Fachinformationszentrum Karlsruhe, Gesellschaft für wissenschaftlich-technische Information mbH, W-7514 Eggenstein-Leopoldshafen 2, unter Angabe der Hinterlegungsnummer CSD-55667, der Autorennamen und des Zeitschriftenzitats angefordert werden.
Darstellung der Bis/(fluorsilyl)amino]di- und -trisulfane 2, 3 sowie des Bis/(fluorsilyl)amino]selans $4: 4.7 \mathrm{~g}(0.02 \mathrm{~mol}) 1$ und $2.5 \mathrm{~g}$ $(0.01 \mathrm{~mol}) \mathrm{S}_{8}$ bzw. $4 \mathrm{~g}(0.05 \mathrm{~mol})$ graues $\mathrm{Se}$ in $70 \mathrm{ml} n$-Hexan $/ 30 \mathrm{ml}$ THF werden unter Rückfluß erhitzt und gerührt. Der Reaktionsfortschritt wird ${ }^{19} \mathrm{~F}$-NMR-spektroskopisch verfolgt. Die Umsetzung mit Schwefel verläuft exotherm und ist nach ca. $2 \mathrm{~h}$ beendet, die mit Selen nach ca. 48 h. 2 und 3 entstehen auch bei unterschiedlich molaren Ansätzen nebeneinander. Destillativ wurden 2 und 3 nicht vollständig voneinander getrennt. Durch Kristallisation aus $n$-Hexan konnte 3 jedoch rein isoliert werden. 4 ist nach der Vakuumdestillation analysenrein.

Tab. 1. Atomkoordinaten $\left(\times 10^{4}\right)$ und äquivalente isotrope Auslenkungsparameter $\left[\mathrm{pm}^{2} \times 10^{-1}\right]$ von 3 .

\begin{tabular}{|c|c|c|c|c|}
\hline & $\mathrm{x}$ & $\mathrm{y}$ & $\mathbf{z}$ & $\mathrm{u}(\mathrm{eq}) *$ \\
\hline$s(1)$ & $8818(1)$ & $319(1)$ & $523(1)$ & $29(1)$ \\
\hline$s(2)$ & $9932(1)$ & $1194(1)$ & $1057(1)$ & $35(1)$ \\
\hline$s(3)$ & $8243(1)$ & $1960(1)$ & $1392(1)$ & $29(1)$ \\
\hline$N(1)$ & $9198(3)$ & $525(1)$ & $-167(1)$ & $27(1)$ \\
\hline$c(5)$ & $8315(4)$ & $1185(2)$ & $-510(1)$ & $34(1)$ \\
\hline$C(51)$ & $7451(5)$ & $747(2)$ & $-990(2)$ & $61(1)$ \\
\hline$c(52)$ & $9381(5)$ & $1825(2)$ & $-755(2)$ & $82(2)$ \\
\hline$C(53)$ & $7205(5)$ & $1632(2)$ & $-131(2)$ & $69(1)$ \\
\hline Si(1) & $10487(1)$ & $-238(1)$ & $-402(1)$ & $26(1)$ \\
\hline $\mathbf{F}(1)$ & $10581(2)$ & $-75(1)$ & $-1086(1)$ & $40(1)$ \\
\hline$c(1)$ & $9726(4)$ & $-1369(2)$ & $-339(1)$ & $35(1)$ \\
\hline$C(11)$ & $9965(5)$ & $-1788(2)$ & $251(1)$ & $60(1)$ \\
\hline$c(12)$ & $10481(5)$ & $-1935(2)$ & $-795(1)$ & $58(1)$ \\
\hline$C(13)$ & $8047(4)$ & $-1357(2)$ & $-469(2)$ & $56(1)$ \\
\hline$c(2)$ & $12461(4)$ & $-30(2)$ & $-143(1)$ & $37(1)$ \\
\hline$C(21)$ & $12783(4)$ & $917(2)$ & $-213(2)$ & $61(1)$ \\
\hline$c(22)$ & $12807(4)$ & $-286(3)$ & $481(1)$ & $64(1)$ \\
\hline$C(23)$ & $13542(4)$ & $-527(2)$ & $-539(2)$ & $61(1)$ \\
\hline$N(2)$ & $7719(3)$ & $1605(1)$ & $2045(1)$ & $26(1)$ \\
\hline$c(6)$ & $6627(3)$ & $870(2)$ & $2083(1)$ & $30(1)$ \\
\hline$c(61)$ & $5689(4)$ & $932(2)$ & $2628(1)$ & $49(1)$ \\
\hline$C(62)$ & $7501(5)$ & $37(2)$ & $2089(2)$ & $56(1)$ \\
\hline$c(63)$ & $5558(4)$ & $884(2)$ & $1575(1)$ & $56(1)$ \\
\hline Si(2) & $8535(1)$ & $2271(1)$ & $2564(1)$ & $25(1)$ \\
\hline $\mathbf{F}(2)$ & $7968(2)$ & $1912(1)$ & $3171(1)$ & $43(1)$ \\
\hline$c(3)$ & $7700(4)$ & $3384(2)$ & $2527(1)$ & $37(1)$ \\
\hline$C(31)$ & $6013(5)$ & $3286(2)$ & $2456(2)$ & $87(2)$ \\
\hline$c(32)$ & $7988(6)$ & $3856(2)$ & $3089(1)$ & $75(2)$ \\
\hline$C(33)$ & $8256(6)$ & $3932(2)$ & $2038(2)$ & $70(2)$ \\
\hline$c(4)$ & $10637(3)$ & $2165(2)$ & $2631(1)$ & $31(1)$ \\
\hline$C(41)$ & $11585(4)$ & $2578(2)$ & $2159(1)$ & $46(1)$ \\
\hline$C(42)$ & $11122(4)$ & $2554(2)$ & $3212(1)$ & $51(1)$ \\
\hline$C(43)$ & $11001(4)$ & $1209(2)$ & $2659(2)$ & $49(1)$ \\
\hline
\end{tabular}

* Äquivalente isotrope $U$ berechnet als ein Drittel der Spur des orthogonalen $U_{i j}$-Tensors

2 : Sdp. $140-150^{\circ} \mathrm{C} / 0.01 \mathrm{mbar}$. Ausb. $1.0 \mathrm{~g} \mathrm{(20 \% )}$ - - MS (FJMessung): $m / z(\%)=528(22)\left[\mathrm{M}^{+}\right], 264(100)\left[1 / 2 \mathrm{M}^{+}\right] .-{ }^{1} \mathrm{H}-$ NMR $\left(\mathrm{CDCl}_{3}\right): \delta=1.17\left[\mathrm{~d}, 9 \mathrm{H}, \mathrm{SiC}\left(\mathrm{CH}_{3}\right)_{3},{ }^{4} J_{\mathrm{HF}}=1.8 \mathrm{~Hz}\right], 1.18$ $\left[\mathrm{d}, 9 \mathrm{H}, \mathrm{SiC}\left(\mathrm{CH}_{3}\right)_{3},{ }^{4} J_{\mathrm{HF}}=1.8 \mathrm{~Hz}\right], 1.40\left[\mathrm{~d}, 9 \mathrm{H}, \mathrm{NC}\left(\mathrm{CH}_{3}\right)_{3},{ }^{5} J_{\mathrm{HF}}=\right.$ $2.3 \mathrm{~Hz}] .-{ }^{13} \mathrm{C}-\mathrm{NMR}\left(\mathrm{CDCl}_{3}\right): \delta=21.71\left(\mathrm{~d},{ }^{2} J_{\mathrm{CF}}=13.3 \mathrm{~Hz}, \mathrm{SiC}\right)$, $22.33\left(\mathrm{~d},{ }^{2} J_{\mathrm{CF}}=13.6 \mathrm{~Hz}, \mathrm{SiC}\right), 28.74\left(\mathrm{~d},{ }^{3} J_{\mathrm{CF}}=0.9 \mathrm{~Hz}, \mathrm{SiCC}_{3}\right), 29.44$ $\left(\mathrm{d},{ }^{3} J_{\mathrm{CF}}=1.3 \mathrm{~Hz}, \mathrm{SiCC}_{3}\right), 31.19\left(\mathrm{~d},{ }^{4} J_{\mathrm{CF}}=4.7 \mathrm{~Hz}, \mathrm{NCC}_{3}\right), 59.80(\mathrm{~d}$, $\left.{ }^{3} J_{\mathrm{CF}}=2.4 \mathrm{~Hz}, \mathrm{NC}\right) .-{ }^{19} \mathrm{~F}-\mathrm{NMR}\left(\mathrm{CDCl}_{3}, \mathrm{C}_{6} \mathrm{~F}_{6}\right): \delta=12.7 .-{ }^{29} \mathrm{Si}-$ NMR $\left(\mathrm{CDCl}_{3}\right): \delta=-6.13\left(\mathrm{~d}, J_{\mathrm{SiF}}=309.7 \mathrm{~Hz}\right.$.)

3: Sdp. $150^{\circ} \mathrm{C} / 0.01 \mathrm{mbar}$. - MS (FJ-Messung): $m / z=560(100)$ $\left[\mathrm{M}^{+}\right] .-{ }^{1} \mathrm{H}-\mathrm{NMR}\left(\mathrm{CDCl}_{3}\right): \delta=1.10\left[\mathrm{~d}, 9 \mathrm{H}, \mathrm{SiC}\left(\mathrm{CH}_{3}\right)_{3},{ }^{4} J_{\mathrm{HF}}=\right.$ $1.2 \mathrm{~Hz}], 1.11\left[\mathrm{~d}, 9 \mathrm{H}, \mathrm{SiC}\left(\mathrm{CH}_{3}\right)_{3},{ }^{4} J_{\mathrm{IFF}}=1.2 \mathrm{~Hz}\right], 1.38[\mathrm{~d}, 9 \mathrm{H}$, $\left.\mathrm{NC}\left(\mathrm{CH}_{3}\right)_{3},{ }^{5} J_{\mathrm{Hr}}=1.8 \mathrm{~Hz}\right] .-{ }^{13} \mathrm{C}-\mathrm{NMR}\left(\mathrm{CDCl}_{3}\right): \delta=21.17(\mathrm{~d}$, $\left.{ }^{2} J_{\mathrm{CF}}=13.8 \mathrm{~Hz}, \mathrm{SiC}\right), 22.85\left(\mathrm{~d},{ }^{2} J_{\mathrm{CF}}=12.9 \mathrm{~Hz}, \mathrm{SiC}\right), 28.75\left(\mathrm{~d},{ }^{3} J_{\mathrm{CF}}=\right.$ 
$\left.1.1 \mathrm{~Hz}, \mathrm{SiCC}_{3}\right), 29.68\left(\mathrm{~d},{ }^{3} J_{\mathrm{CF}}=1.3 \mathrm{~Hz}, \mathrm{SiCC}_{3}\right), 32.18\left(\mathrm{~d},{ }^{4} J_{\mathrm{CF}}=\right.$ $\left.4.5 \mathrm{~Hz}, \mathrm{NCC}_{3}\right), 59.10\left(\mathrm{~d},{ }^{3} J_{\mathrm{CF}}=2.4 \mathrm{~Hz}, \mathrm{NC}\right) .-{ }^{19} \mathrm{~F}-\mathrm{NMR}\left(\mathrm{CDCl}_{3}\right.$, $\left.\mathrm{C}_{6} \mathrm{~F}_{6}\right): \delta=10.8 .-{ }^{29} \mathrm{Si}-\mathrm{NMR}\left(\mathrm{CDCl}_{3}\right): \delta=-4.30\left(\mathrm{~d}, J_{\mathrm{SiF}}=\right.$ $308.4 \mathrm{~Hz}$ ).

4: Sdp. $160^{\circ} \mathrm{C} / 0.01$ mbar. - MS (FJ-Messung): $m / z=544$ (3) $\left[\mathrm{M}^{+}\right], 311(100) .-{ }^{1} \mathrm{H}-\mathrm{NMR}\left(\mathrm{CDCl}_{3}\right): \delta=1.03[\mathrm{~d}, 18 \mathrm{H}, \mathrm{Si}-$ $\left.\mathrm{C}\left(\mathrm{CH}_{3}\right)_{3},{ }^{4} J_{\mathrm{HF}}=1.1 \mathrm{~Hz}\right], 1.78\left[\mathrm{~d}, 9 \mathrm{H}, \mathrm{NC}\left(\mathrm{CH}_{3}\right)_{3},{ }^{5} J_{\mathrm{HF}}=0.9 \mathrm{~Hz}\right]$. $-{ }^{19} \mathrm{~F}-\mathrm{NMR}\left(\mathrm{CDCl}_{3}, \mathrm{C}_{6} \mathrm{~F}_{6}\right): \delta=9.0 .-{ }^{29} \mathrm{Si}-\mathrm{NMR}\left(\mathrm{CDCl}_{3}\right): \delta=$ $-42.60\left(\mathrm{~d}, J_{\mathrm{SiF}}=274.6 \mathrm{~Hz}\right)$.

$$
\begin{array}{ll}
\mathrm{C}_{24} \mathrm{H}_{54} \mathrm{~F}_{2} \mathrm{~N}_{2} \mathrm{SeSi}_{2} \text { (543.5) } & \text { Ber. C } 53.04 \text { H } 9.94 \\
& \text { Gef. C } 52.43 \text { H } 9.36
\end{array}
$$

CAS-Registry-Nummern

1: $58802-37-2$ / 2: 135740-63-5 / 3: 135740-64-6 / 4: 135740-65-7 $\mathrm{S}_{8}: 10544-50-0 / \mathrm{Se}: 7782-49-2$
1) O. J. Scherer, Angew. Chem. 81 (1969) 871; Angew. Chem. Int. Ed.Engl. 8 (1969) 861

2) M. Björgvinsson, H. W. Roesky, F. Pauer, D. Stalke, G. M. Sheldrick, Inorg. Chem. 29 (1990) 5140.

${ }^{3)}$ G. Schubert, G. Kiel, G. Gattow, Z. Anorg. Allg. Chem. 575 (1989) 129.

4) U. Klingebiel in Silicon Chemistry (J. Y. Corey, E. R. Corey, P. P. Gaspar, Hrsg.), Bd. 31, S. 337, Ellis Horwood Ltd., Chichester, England, 1988.

5) S. Vollbrecht, U. Klingebiel, D. Schmidt-Bäse, Z. Naturforsch. Teil B 46 (1991) 709.

6) S. Walter, U. Klingebiel, D. Schmidt-Bäse, J. Organomet. Chem., im Druck.

7) C. Pauling, Die Natur der chemischen Bindung, VCH Verlagsgesellschaft, Weinheim 1968.

${ }^{8)}$ G. M. Sheldrick, Acta Crystallogr., Sect. A, 46 (1990) 467.

9) G. M. Sheldrick, SHELX-76, erweiterte Version, Universität Cambridge 1976.

$[224 / 91]$ 